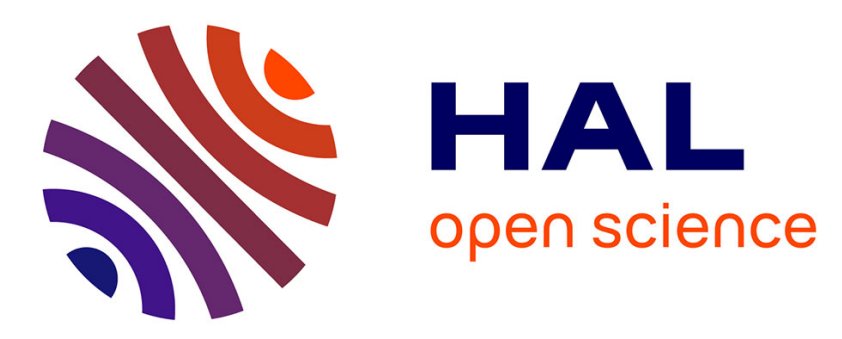

\title{
Creep investigations on adhesively bonded fasteners developed for offshore steel structures
}

Ethel Djeumen, Sylvain Chataigner, Romain Créac'Hcadec, Quentin

Sourisseau, Marie-Odette Quemere, Jean-Philippe Court, Firas Sayed

\section{To cite this version:}

Ethel Djeumen, Sylvain Chataigner, Romain Créac'Hcadec, Quentin Sourisseau, Marie-Odette Quemere, et al.. Creep investigations on adhesively bonded fasteners developed for offshore steel structures. Marine Structures, 2020, 69, 14 p. 10.1016/j.marstruc.2019.102660 . hal-02431022

\section{HAL Id: hal-02431022 \\ https://hal.science/hal-02431022}

Submitted on 7 Jun 2021

HAL is a multi-disciplinary open access archive for the deposit and dissemination of scientific research documents, whether they are published or not. The documents may come from teaching and research institutions in France or abroad, or from public or private research centers.
L'archive ouverte pluridisciplinaire HAL, est destinée au dépôt et à la diffusion de documents scientifiques de niveau recherche, publiés ou non, émanant des établissements d'enseignement et de recherche français ou étrangers, des laboratoires publics ou privés. 


\section{Creep investigations on adhesively bonded fasteners developed for} 2 offshore steel structures

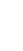

E. Djeumen ${ }^{1}$, S. Chataigner ${ }^{1}$, R. Créac'hcadec ${ }^{2}$, Q.Sourisseau ${ }^{1}$, M.O. Quéméré ${ }^{3}$, J.P. Court ${ }^{3}$, F. Sayed ${ }^{3}$

${ }^{1}$ IFSTTAR, Laboratoire Structure Métallique à Câble (SMC), Département MAST, Route de Bouaye, 44344 Bouguenais

Corresponding author: sylvain.chataigner@ifsttar.fr

2 ENSTA Bretagne, UMR CNRS 6027, IRDL, F-29200 Brest, France

${ }^{3}$ COLD PAD, 130 Rue de Lourmel, 75015 Paris

\section{Abstract}

Fastening or reinforcement onto an existing steel structure may create constraints such as the use of hot works (i.e. welding) or loss of weathertightness (i.e. drilling holes and bolting). In order to limit these constraints, there may be a strong interest in using adhesively bonded fasteners. Such a solution requires however strong justification especially regarding long-term properties. This paper presents the results of experimental and numerical investigations realized on the creep behaviour of such bonded fasteners developed for offshore steel structures. Experimental investigations were conducted at room temperature following a precise and repeatable implementation protocol. The results of the various investigations revealed a non-linear evolution of creep displacements according to a Burger law. Numerical modelling has therefore been developed on the basis of Burger's law. A preliminary static study aimed at validating the geometric model and analyzing the initial stress state in the adhesive. The creep study was then carried out to compare numerical results to experimental ones. Although additional investigations are needed, numerically determined creep displacements demonstrated a good consistency with the experimental investigations.

Keywords: Creep, Adhesive bonding, Fastener, Modelling, Experimental investigations. 


\section{Introduction}

Steel structures in service, especially in harsh environments, may be locally affected by fatigue and corrosion and may therefore need reinforcement or repair. In addition, most often there is some need during the structure service life to connect additional equipment, secondary structures or distribution systems. In the case of steel structures, this is carried out using bolted or welded assembly that may be detrimental regarding operation issues (e.g. hot works such as welding in an explosive environment) and may induce heavy on-site constraints for the structure exploitation. In regards with these issues, adhesive bonding solution may appear as a good solution, provided that the robustness and the durability of the assembly is confirmed. The firm Cold Pad, Paris, France is currently developing an adhesively bonded fastener to provide such a solution for offshore applications [1]. They are also developing an overall solution that includes an application disposal ensuring precise control of the adhesive joint quality during application, and water tightness of the joint to prevent any durability issue linked to wet environment [2] (Figure 1).

Durability of adhesively bonded joint covers several damage mechanisms that may be induced by the environment or the applied load [3]. In the case of the studied bonded fasteners, as water tightness is ensured, creep and fatigue appear the main durability issues that needs to be addressed [4-5]. It was decided to first investigate creep behaviour without taking into account fatigue creep phenomena that may arise [6]. Most often, experimental creep studies of adhesively bonded joints are carried out at the scale of the adhesive alone [7]. Yet, it is well known that stress states at full scale of the bonded assembly are strongly non-uniform [8]. It was thus decided for this study to realize experimental investigations at the scale of the bonded fastener, and to develop in parallel a finite element model. This model takes into account the creep behaviour of the adhesive in order to be able to visualize the internal stress state of the joint during the test and to compare some local measurements with numerical results.

A short literature review on the main available creep models will first be introduced. Then, the developed experimental investigations will be described. The aim was to investigate the two 
main loadings that may encounter the developed bonded fasteners: tension loading perpendicular to the bonded surface, and shear loading parallel to the bonded surface. After a presentation of the obtained results, the developed finite model will be introduced and the obtained results analysed. The last part will be dedicated to the comparison of the numerical model with the experimental results.

2. Literature review on creep modelling

Creep is the response of a material to a constant stress [9]. The creep is characterized by three stages as illustrated in Figure 2 delimited by the evolution of the strain rate: primary stage with a decreasing strain rate, secondary stage with a constant strain rate and tertiary stage with an increasing of strain rate until the failure.

Mathematically, the creep strain $\varepsilon(t)$ should be noted in the form of equation (1) [9].

$\varepsilon(\mathrm{t})=\sigma^{0} \mathrm{~J}\left(\mathrm{t}, \mathrm{T}, \sigma^{0}\right)$

$J$ being creep compliance that depends mainly on time $t$, temperature $T$ and applied stress $\sigma^{0}$ [10].

Two main categories of modelling strategies exist in the literature: the empirical or semiempirical approaches and the rheological ones. Main models will be described in the two following parts.

\subsection{Semi-empirical models}

Various mathematical forms have been empirically proposed to describe the mechanical behaviour under creep loading. The simplest is a linear function of the stress but non-linear (logarithmic for example) when depending on time. The most commonly used expression of creep compliance is a power law given by equation (2) [11-13].

$J(t)=J_{0}+J_{1} t^{n}$ 
where $\left(J_{0} ; J_{1} ; n\right)$ are material parameters of the model. Other empirical models exist. An example is that of Bailey Norton which allows to take into account the nonlinear behaviour and the influence of the temperature. The expression of creep compliance is then given by equation (3) [9].

$\mathrm{J}(\mathrm{t}, \sigma, \mathrm{T})=\mathrm{C}_{0} \sigma^{\mathrm{C} 1} \mathrm{t}^{\mathrm{C} 2} \exp \left(-\mathrm{C}_{\mathrm{T}} / \mathrm{T}\right)$

$\left(C_{0} ; C_{1} ; C_{2} ; C_{T}\right)$ being the material constants of the model.

Another example of a nonlinear model is given by equations (4) [13]. This empirical model is based on the fact that increasing the stress level has the effect of reducing the relaxation time $t_{0}$.

$\mathrm{J}(\mathrm{t}, \sigma)=\mathrm{J}_{0} \exp \left(-\mathrm{t} / \mathrm{t}_{0}\right)^{\mathrm{m}}$

$\mathrm{t}_{0}=A \exp \left(-\alpha \sigma_{0}^{2}\right)^{\mathrm{m}}$

where $(A ; \alpha)$ are material parameters of the model.

\subsection{Rheological Models}

The creep behaviour can also be described by a rheological approach with basic mechanical systems. Figure 3.a and Figure 3.b below show the rheological formulation and the representation of elasticity by the spring $(E)$ and the viscosity by the dashpot $(\eta)$, where $\sigma$ is the stress in the system and $\varepsilon$ is the strain. Kelvin-Voigt's model combines spring and dashpot in parallel (Figure 3.c). It is a solid model at infinity [9]. The expression of creep compliance is given by equation (5).

$J(t)=1 / E[1-\exp (E / \eta . t)]$

$\eta / E$ being the characteristic time of the model.

The creep response of a material for the Kelvin-Voigt model is shown in Figure 3.c below. We find that this model does not predict the instantaneous elasticity $(\mathrm{J}(0)=0)$. It only illustrates the first stage of creep. 
The Maxwell model itself consists of a spring and a dashpot mounted in series (Figure 3.d). It is a fluid model at infinity [9]. The expression of creep compliance is:

$$
J(t)=1 / E+t / n
$$

where $E$ is the Young's modulus and $\eta$ the viscous parameters.

The creep response of a material for the Maxwell model is shown in Figure 3.d below. This model brings out the second stage of creep.

The Burger model is obtained by associating in series the Kelvin-Voigt model and the Maxwell model. This model shows the first two stages of creep (Figure 3.e). Creep compliance is obtained by summing up the compliances of the Kelvin-Voigt and Maxwell models (equation 7) [4].

$J(t)=1 / E_{1}+t / \eta_{1}+1 / E_{2}\left[1-\exp \left(-E_{2} / \eta_{2} \cdot t\right)\right]$

where $\left(E_{1} ; E_{2}\right)$ are the spring parameters and $\left(\eta_{1} ; \eta_{2}\right)$ the viscous ones.

\section{3. $3 D$ creep}

The concept of creep can be generalized in 3D. The creep compliance $\boldsymbol{J}$ is in this case a tensor of order 4 . The response to 3D creep is of the form of equation (8) [9].

$\boldsymbol{\varepsilon}(\mathrm{t})=\mathbf{J}: \boldsymbol{\sigma}^{0}$ In the hypothesis of an isotropic linear viscoelastic behaviour, the relation (8) takes the form of equation (9) [9].

$\varepsilon(t)=(1+v(t)) J(t) \sigma^{0}-v(t) J(t) \operatorname{tr}\left(\sigma^{0}\right) I$ where $J(t)$ and $v(t)$ are respectively the uniaxial creep compliance and the Poisson's ratio determined from a simple tensile creep experiment carried out in any direction. In general, the Poisson's ratio doesn't depend on time, $v(t)=v[14]$. 
142 It was decided to focus in the presented work on the first two stages of creep relying on the

143 use of the presented Burger rheological model and to make a hypothesis of an isotropic 144 behaviour.

3. Experimental investigations on adhesively bonded fasteners.

The led investigations have been carried out at full scale of the developed adhesively bonded fasteners. Preliminary quasi-static tests have been realized to determine the ultimate capacities of the assemblies for two solicitations: tension loading (perpendicular to the bonded surface) and shear loading (parallel to the bonded surface). Then, creep investigations have been carried out for the two solicitations.

\subsection{Description of the tested specimens}

The tested steel fasteners have been developed by Cold Pad, Paris, France and are called CClaw $_{\mathrm{TM}}$ [1]. Two different fasteners were tested: one dedicated to a shear dominant solicitation mode: fastener $\mathrm{S}$, and the other one dedicated to a tension dominant solicitation mode: fastener $\mathrm{T}$. A photo of both studied fasteners $\mathrm{T}$ and $\mathrm{S}$ is given in Figure 4. Both consists in a circular steel plate with a connected steel stud (i.e. thread rod). The circular steel plate geometry has been adapted to the applied load.

These fasteners have been bonded to square $20 \mathrm{~mm}$ thick steel plates. The studied adhesive is a commercial methacrylate adhesive whose main characteristics are given in table 1 where E are respectively the Young's modulus, $R T$, the Tensile strength and $T g$, the Glass transition temperature. The surface preparation has been carried out in agreement with the adhesive specifications.

Two specific test settings have been developed to be able to apply the selected solicitation using a hydraulic test machine both in quasi-static and creep situations (Figure 5). 
The tensile test was performed with the tensile fastener bonded to a $20 \mathrm{~mm}$ thick plate bolted to a tensile frame. The assembly was connected to a hydraulic test machine via the tensile frame for the upper grip and a connection sleeve for the lower grip as shown in Figure 6. Alignment of the frame and the connection sleeve was essential to macroscopically ensure a load in pure tension and limit the cleavage of the assembly. The loading was applied at a speed of $1.2 \mathrm{kN} / \mathrm{s}$. Three LVDT sensors placed at $120^{\circ}$ measured out-of-plane displacements during the test as shown in Figure 6. It allowed obtaining indication on the symmetry of the test, but also on the long-term behaviour during creep tests.

\subsection{Shear test protocol}

The shear test in principle was identical to that in tension. The shear fastener was bonded to a $20 \mathrm{~mm}$ thick plate bolted to the shear frame. Figure 7 presents the assembly used for the realization of shear tests. The assembly was connected to the loading machine via a shear frame for the upper grip and the connection plate for the lower grip. For the same reason as in tension, the alignment of the shear frame and the connection plate had to be achieved to limit cleavage of the assembly. Loading was done at the same speed of $1.2 \mathrm{kN} / \mathrm{s}$. A sensor makes it possible to measure the displacement in the plane (sensor 1) and three sensors placed at $120^{\circ}$ made it possible to measure the out-of-plane displacement (sensors $2,3,4$ ) during the test as illustrated in Figure 7.

\subsection{Preliminary quasi static investigations}

For both solicitations, preliminary quasi static investigations up to failure were carried out to determine the ultimate capacities of the bonded assemblies. Three tests for each configuration were realized at $25{ }^{\circ} \mathrm{C}$. Similar capacities were obtained for both configurations around 100 $\mathrm{kN}$, and the obtained variations were 4 and $12 \%$ for respectively the shear and tension tests. The results allowed obtaining ultimate capacities used to settle the applied load during the 
creep investigations. The average ultimate capacity in tension will be denoted $\mathrm{T}$ and the one in shear will be denoted S.

\subsection{Results of tensile creep tests}

It was decided to carry out two creep tests in tension at an applied load corresponding respectively to $59 \%$ and $69 \%$ of the ultimate load capacity T. Figure 8.a and Figure 8.b shows the evolution of the experimentally measured out-of-plane displacements for the tensile creep tests for these two respective loads. The $59 \%$ test lasted 10 hours 36 minutes before being interrupted. Only the first two creep stages are visible in Figure 8 . The $69 \%$ test lasted 3 hours 16 minutes before failure occurred. The displacement measured by the sensor 3 made it possible to distinguish the three creep stages. The first stage lasted about 30 minutes and the second stage 2 hours before the final stage started (stage 3). Interestingly, in both cases, it can be noticed that measured displacements are highly asymmetric. There seems to be a localisation of the phenomenon. Obviously, creep phenomenon was more pronounced when the applied load is higher.

\subsection{Results of creep shear tests}

The shear creep tests were carried out for closed values of the percentage of the applied load in comparison with the ultimate load capacity: 59 and $67 \%$ of the ultimate load capacity in shear S. Figure 9.a and Figure 9.b shows the results of the measured displacements for these two shear creep tests. Only two displacements are presented: sensor 1 represents the displacement in the direction parallel to the bonded surface, sensor 3 represents the peel displacement in the extremity of the assembly. The sensors 2 and 4 measured very low displacements and are therefore not plotted here.

The test at $59 \%$ of the ultimate load capacity in shear S lasted 15 hours before being interrupted. Only the first two creep stages were measured. The in-plane displacement of the shear fastener measured by the sensor 1 was $221 \mu \mathrm{m}$ ( 5 times the static displacement during 
the loading). The out-of-plane displacement of the fastener measured by the sensor 3 has reached $74 \mu \mathrm{m}$ (6 times the static displacement). Only the first two stages were measured.

The test at $67 \%$ of the ultimate load capacity S lasted 25 minutes before failure of the assembly for an in-plane displacement measured by sensor 1 equal to $380 \mu \mathrm{m}$ and an out-of-plane displacement (sensor 3) of $230 \mu \mathrm{m}$. The three creep stages were measured in this case.

\section{Modelling of the tests}

\subsection{Development of models}

For both solicitations, finite element analyses were carried out using the commercial Software Abaqus, Paris, France.

To model the tensile test, it was possible to use a 2D axisymmetric model (Figure 10) by making the assumption that the bolted zones were sufficiently far from the adhesive layer. To further simplify, the assumption was made that the support parts (tensile frame, sleeve) were dimensionally stable and did not need to be taken into account in the model. The behaviours of the adhesive, the plate and the tensile fastener were assumed to be isotropic linear elastic.

In a bonded assembly, the accuracy of the stress state is dependent on the number of elements in the thickness of the adhesive [15]. It was thus decided to lead a preliminar study on the influence of the mesh on the obtained stress states for each studied configuration. Linear rectangular elements were used and chosen as close as possible to square elements. "Cleaned" edges as advised in [15] were modelled for the free end of the adhesive layer to decrease singularity effects in this location. The mesh size was kept constant for the whole model and controlled by the numbers of elements in the adhesive layer. The convergence study investigated 3,6 and 12 elements in the thickness mesh. The results in the case of the 2D modelling of the tensile test are given in figure 11. It can be observed that stress states in the middle of the adhesive are similar for the 6 and 12 elements in the thickness configurations. 

obtain representative results while limiting the needed calculation time.

In order to validate the hypothesis made for the 2D axisymmetric model, a 3D model presented in Figure 12 was also realized. Similar mesh configuration to the 2D model were adopted. The two planes of symmetry of the problem allowed reducing the model to only a quarter of the global geometry (Figure 10).

On the whole, the assumptions made for the shear calculation were the same as in tension. Only the plate, the adhesive and the non-cylindrical part of the fastener were modelled. It was necessary to use a 3D model as the loading was not axisymmetric. The 3D shear model had a plane of symmetry shown in Figure 13 . This plane of symmetry made it possible to model only half of the total geometry. The boundary conditions used for the shear calculation are shown in Figure 13.

\subsection{Study of stress distribution and $2 D / 3 D$ comparison}

As preliminary investigations, the modelling was realized in elastic situation taking into account the obtained average quasi static ultimate capacities for both solicitations. For both cases, several stresses are studied: shear (SXY) and peel stresses (SYY), von Mises equivalent stress (SVM) and hydrostatic pressure (p).

For the case of tension load, Figure 14 presents the normalised stress states (in comparison with the ultimate von Mises stress) along the normalised bonded length (on one radius, as the assembly is axisymmetric). For each studied stress component, three positions in the adhesive layer were studied: top of the adhesive closed to the fastener, middle of the adhesive and bottom of the adhesive closed to the steel plate.

It can be observed that the position in the adhesive thickness does not seem to have a great influence on the studied results. In addition, it can be highlighted that although the macroscopically applied load is a tensile stress, the stress state in the adhesive is not simple. 
275 The multiaxial stress state in the adhesive is explained by the non-regular geometry of the

276 tensile fastener. In the middle of the adhesive layer, there seems to be mostly tensile stresses

277 in the adhesive, whereas, far from the centre, shear stress seems to be predominant.

278 Interestingly, the geometry of C-Claw ${ }_{\mathrm{TM}}$ seems to avoid obtaining stress concentrations at the

279 free edges of the assembly in tension.

280 In order to verify the 2D assumption, a comparison was carried out between $2 \mathrm{D}$ results and

$2813 \mathrm{D}$ results. In the 3D model, as the model is not axisymmetric anymore, two directions were studied (Figure $15 \mathrm{a}$ ). The results in 2D and 3D are very similar as shown in Figure $15 \mathrm{~b}$, thus validating the assumptions made for the calculation in $2 \mathrm{D}$.

For the case of the shear load, the results in terms of normalised stresses (relatively with the maximum von Mises stress) along the main diameter (oriented along the load axis) are given in Figure 16. Again, there is no large difference between the different studied adhesive thickness locations. The obtained stress state is also multiaxial with a non-uniform shear stress and an equilibrated peel stress profiles (due to the applied moment). Interestingly, it can also be checked that the geometry of $\mathrm{C}-\mathrm{Claw}_{\mathrm{TM}}$ seems to avoid obtaining stress concentrations at the free edges of the assembly in shear.

\subsection{Determination of creep parameters from measured displacements}

The creep law used to model the creep behaviour of the adhesive is Burger's law. This is the most used law for polymeric materials. Moreover, the observation of the evolution of displacements measured during creep tests seems to be rather close to the Burger model.

The identification of Burger's law parameters is done by hand for both assemblies assuming a uniaxial tensile stress for tensile creep test and uniaxial shear stress for shear creep test. The used stress for identification is the average tensile and shear stress along the adhesive respectively for tensile and shear creep test. The obtained parameters are given in the table 2. The model is applied to the adhesive material in Abaqus software using a subroutine especially developed for the application. 


\subsection{Study of the von Mises stress distribution during creep}

Figure 17 illustrates the phenomenon of stress redistribution for the tensile model that occurs due to the initial non-uniform distribution of stresses along the adhesive. Figure $17 \mathrm{~b}$ represents the von Mises stress state along the radius of the bonded surface during creep. Figure $17 \mathrm{c}$ shows the average tensile displacement during creep and the localization of the three studied time moments. Interestingly, the stress redistribution with time allows decreasing stress concentration effect, and more particularly the ultimate von Mises stress by $23 \%$.

Figure 18 illustrates the phenomenon of stress redistribution in the case of the shear test. This redistribution was less pronounced in shear than in tension because, initially, the distribution is more uniform in shear than in tension. The maximum von Mises stress after creep was reduced by $14 \%$.

\section{Comparison of measured displacements / model displacement}

\subsection{Tension load}

Figure 19 shows the comparison between the mean creep displacement measured during the tests (mean displacement of the three sensors placed at $120^{\circ}$ ) and the creep displacement determined numerically for the two tested loads. The differences between experimental and numerical curves were negligible. It was possible to model with good accuracy the creep displacements for the first two stages at different levels of loads. Yet, it must be highlighted that it was necessary to modify Burger's parameters and that they seem consequently to be dependent on the load level.

\subsection{Shear load}

Figures 20 and 21 represent the comparison of experimental and numerical results of out-ofplane (sensor 3, Figure 20.b and Figure 21.b) and in-plane (sensor 1, Figure 20.a and Figure 21.a) displacements for shear creep tests with the two studied load levels. The difference between the experimental curve and the numerical one for the creep displacement in the plane was negligible (Figure 20.a and Figure 21.a). Again, the used modelling approach seems to 
be well suited to the modelling of the creep of the assembly. Similarly, to the tension case, the

Burger parameters had to be modified indicating a dependency of these parameters to the applied load. As far as out-of-plane displacements were concerned, there seemed to be large differences between the experimental measures and the model (Figure 20.b and Figure 21.b). There is therefore a need to improve the use model especially regarding isotropy hypothesis.

\subsection{Discussion on future developments}

The presented work allowed settling a modelling approach of the creep behaviour of adhesively bonded fasteners and checking its adequacy for the first two stages of creep. On the basis of the presented work, additional experimental investigations will be carried out with different load levels for both configurations to assess the load dependency of the creep model parameters. This should also improve the proposed modelling approach taking into account for example anisotropy. Additional developments will then be carried out to include stage 3 modelling approach in order to fully understand creep mechanisms and propose design guidelines and state indicators of such technical solution.

\section{Conclusions}

If the interest in using adhesively bonded fasteners exists for steel structures, there have been few studies presenting experimental investigations led at the scale of the fastener. The presented study is one of those and aimed at initiating investigations on the creep behaviour of such fastener.

The presented work investigated C-Claw ${ }_{\mathrm{TM}}$ solution developed by Cold Pad, Paris, France with a methacrylate adhesive system [1]. After a specific development on the test protocol, two main load situations that may be encountered on site were studied: tension and shear. Preliminary investigations allowed determining the ultimate capacities and the repeatability of the assembly process.

A modelling work was then carried out using commercial finite element software Abaqus, Paris, France with specific development dedicated to the integration of Burger's isotropic creep 
model. This work aimed at first in analysing the initial stress state in both load situations (tension and shear). It was proved that for both cases, stress is not uniform and is actually composed of peel and shear components (or pressure and von Mises stresses) depending on the fastener's geometry and the applied load.

Creep modelling was then carried out with an initial determination of Burger's parameters from the obtained experimental results. A good agreement was obtained between the measured average main displacements (out of plane in the case of tension and in plane in the case of shear), and the numerically obtained ones provided that Burger's parameters were dependent on the applied load. The stress redistribution during creep was also highlighted for both load situations during stages 1 and 2 . This in agreement with other studies led on different applications [16-19]. In the case of shear load, a closer study of the measured displacements allowed determining large differences for the out-of-place displacement. This seems to indicate that there may be an anisotropic behaviour of the adhesive that is not currently taken into account in the modelling. Additional investigations are under progress to improve Burgers parameters identification, and study the possible implementation of anisotropic creep behaviour for stages 1 and 2 . There is also a strong need for a model that would consider stage 3 to be able to model entirely creep process of such a bonded assembly [20].

\section{References}

[1] Court JP, Bonded assembly and bonding method, Patent WO2017089668 (A1). 2017.

[2] Hashim SA. Adhesive bonding of thick steel adherends for marine structures. Mar Struct. 1999; 12:405-423. https://doi.org/10.1016/S0951-8339(99)00029-5.

[3] Kinloch AJ. The science of adhesion - Part 2: Mechanics and mechanisms of failure. J of Mat Sci 1982; 17:617-651.

[4] Godzimirski J, Roskowicz M. Numerical analysis of long-lasting strength of adhesive bonds. Adv in Manuf Sci and Tech 2004; 28(1):67-83. 
[6] Al-Ghamdi A. Fatigue and creep of adhesively bonded joints. Phd Thesis, Loughborough

[7] Broughton W. Creep testing of adhesive joints analysis of creep rupture data. Technical report, NPL; 1999.

[8] Cognard JY, Creach'cadec R, Maurice J, 2011. Numerical analysis of the stress distribution in single-lap shear tests under elastic assumptions - application to the optimisation of the mechanical behaviour. Intern $J$ of Adhes and Adhes 2011, 31:715-724. https://doi.org/10.1016/j.ijadhadh.2011.07.001.

[9] Salençon J. Viscoélasticité pour le calcul des structures, l'École polytechnique Edition. Presse de l'École Nationale des Ponts et Chaussée, Paris ; 2016.

[10] Billon N, Bouvard JL. Propriétés et comportement mécanique des polymères thermoplastiques. Techniques de l'Ingénieur, AM3115; 2015.

[11] Penny RK, Mariott DL. Design for creep, McGraw-Hill, London; 1971.

[12] Da Silva LFM, Oschner A, Adams RD. Handbook of adhesion technology, Springer; 2011.

[13] Dean G, Urquhart J, Roberts S. Modelling creep in toughened epoxy adhesives for finite element analysis. Proceedings of Ninth Intern conf on the sci and tech of adhes and adhes, Oxford; 2005.

[14] Thai M. Modélisation micromécanique et simulation numérique du fluage des bétons avec prise en compte de l'endommagement et des effets thermo-hydriques. Ph.D. thesis, Université Paris-Est; 2012.

404

[15] Cognard J, Sohier L, Creac'hcadec R, Lavelle F, Lidon N. Influence of the geometry of 405 coaxial adhesive joints on the transmitted load under tensile and compression loads. Intern $\mathrm{J}$ of Adhes and Adhes 2012; 37:37-49. https://doi.org/10.1016/j.ijadhadh.2012.01.013.

407 [16] Roy S, Reddy JN, 1986. Nonlinear viscoelastic analysis of adhesively bonded joints. 408 Research report N0 VPI-E-86-28; Virginia Tech centre for adhesion science; 1986. 
409 [17] Su N, Mackie RI. Two-dimensional creep analysis of structural adhesive joints. Intern J of 410 adhes and adhes 1992; 13(1): 33-40. https://doi.org/10.1016/0143-7496(93)90006-U.

411 [18] Houhou N, Benzarti K, Quiertant M, Chataigner S, Flety A, Marty C. Analysis of the non412 linear creep behaviour of FRP-concrete bonded assemblies. J of Adhes and Sci Tech 2014; $413 \quad 29(14-15): 1345-1366$.

414 [19] Reza A, Shishesaz M, Naderan-Tahan K, 2014. The effect of viscoelasticity on creep 415 behaviour of double-lap adhesively bonded joints. Lat Am J of solids and struct 2014; 11:3541650.

417 [20] Zehsaz M, Vakili-Tahami F, Saeimi-Sadigh MA, 2014. Parametric study of the creep failure 418 of double lap adhesively bonded joints. Mat and Des 2014; 64:520-526. 
Fig. 1: Photo of the solution developed by Cold Pad (application disposal on the left and adhesively bonded fastener on the right).

Fig. 2: Creep stages.

Fig. 3: Rheological creep models.

Fig. 4: Photo of studied $\mathrm{C}-\mathrm{Claw}_{\mathrm{TM}}$ steel fastener $\mathrm{T}$ on the left and $\mathrm{S}$ on the right.

Fig. 5: Scheme of the two tested solicitation modes: Tension and Shear loadings.

Fig. 6: Protocol of the tensile creep test.

Fig. 7: Protocol of shear creep test.

Fig. 8: Measured displacements during tensile creep investigations at $T=25^{\circ} \mathrm{C}$

Fig. 9: Creep shear at $\mathrm{T}=25^{\circ} \mathrm{C}$.

Fig. 10: Scheme of the studied 2D axisymmetric model.

Fig. 11: Stress results obtained through finite element along the radius in the middle of the adhesive layer for different mesh sizes in tension.

Fig. 12: 3D model in tension.

Fig. 13: Boundary conditions for the shear model.

Fig. 14: Stress state for 2D axisymmetric model in tension.

Fig. 15: Comparison of the 2D and 3D model in tension.

Fig. 16: Stress state along the adhesive for the shear model.

Fig. 17: Redistribution of Von Mises stress for tensile model.

Fig. 18: Redistribution of Von Mises stress for the shear model.

Fig. 19: Experimental and numerical comparison of out-of-plane displacement for tensile creep test at $25^{\circ} \mathrm{C}$.

Fig. 20: Experimental and numerical comparison of creep displacements (in-plane and outof-plane) for shear test at $59 \%$ and $25^{\circ} \mathrm{C}$.

Fig. 21: Experimental and numerical comparison of creep displacements (in-plane and outof-plane) for shear test at $67 \%$ and $25^{\circ} \mathrm{C}$. 


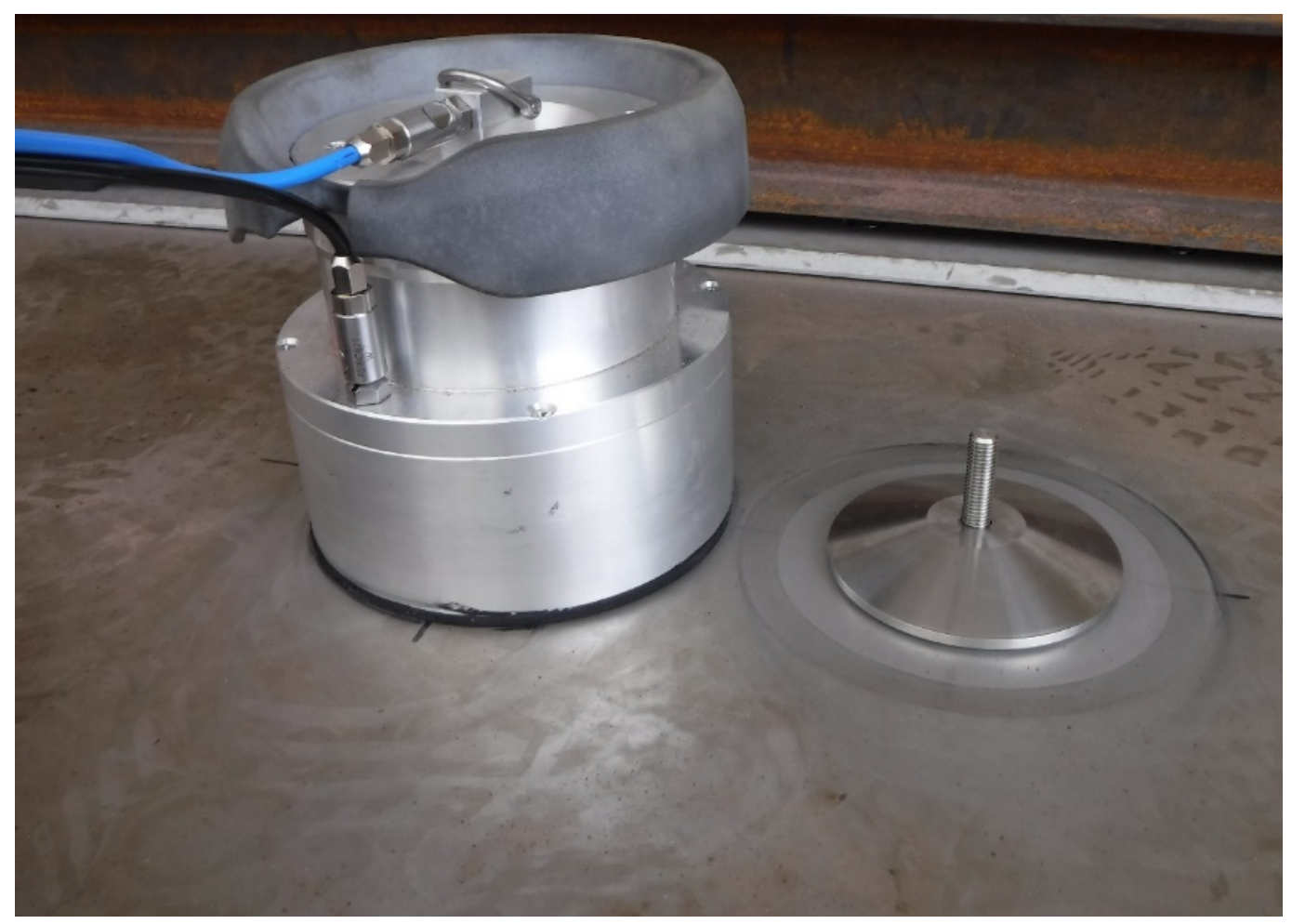

Fig. 1: Photo of the solution developed by Cold Pad (application disposal on the left and adhesively bonded fastener on the right) 


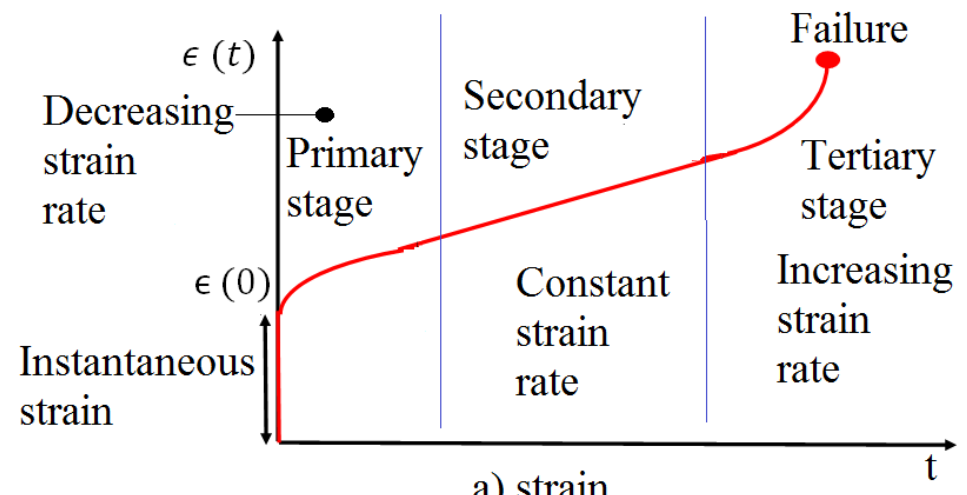

a) strain

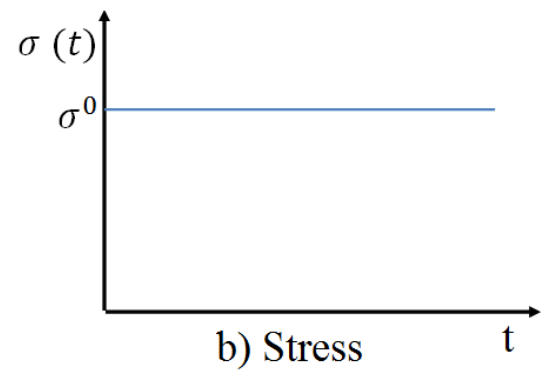

Fig. 2: Creep stages. 
$\leadsto-\infty \square^{\prime} \Rightarrow \sigma=E \varepsilon$

a) Elasticity: spring

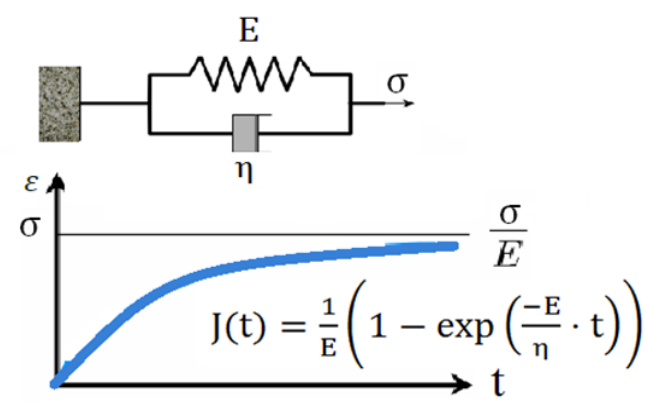

c) Kelvin-Voigt model

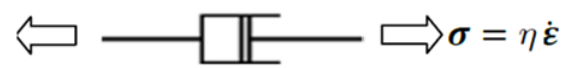

b) Viscosity: Dashpot
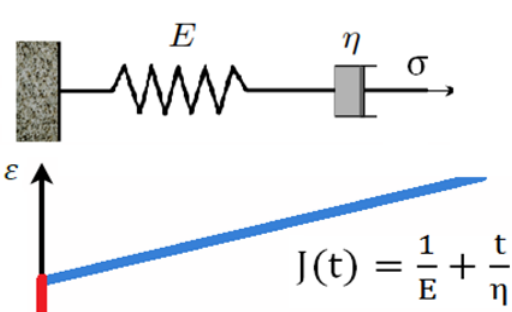

Instantaneous elasticity

d) Maxwell model

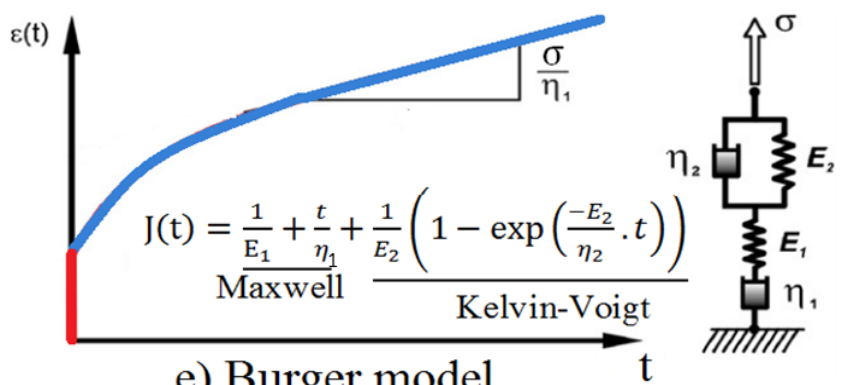

Fig. 3: Rheological creep models. 


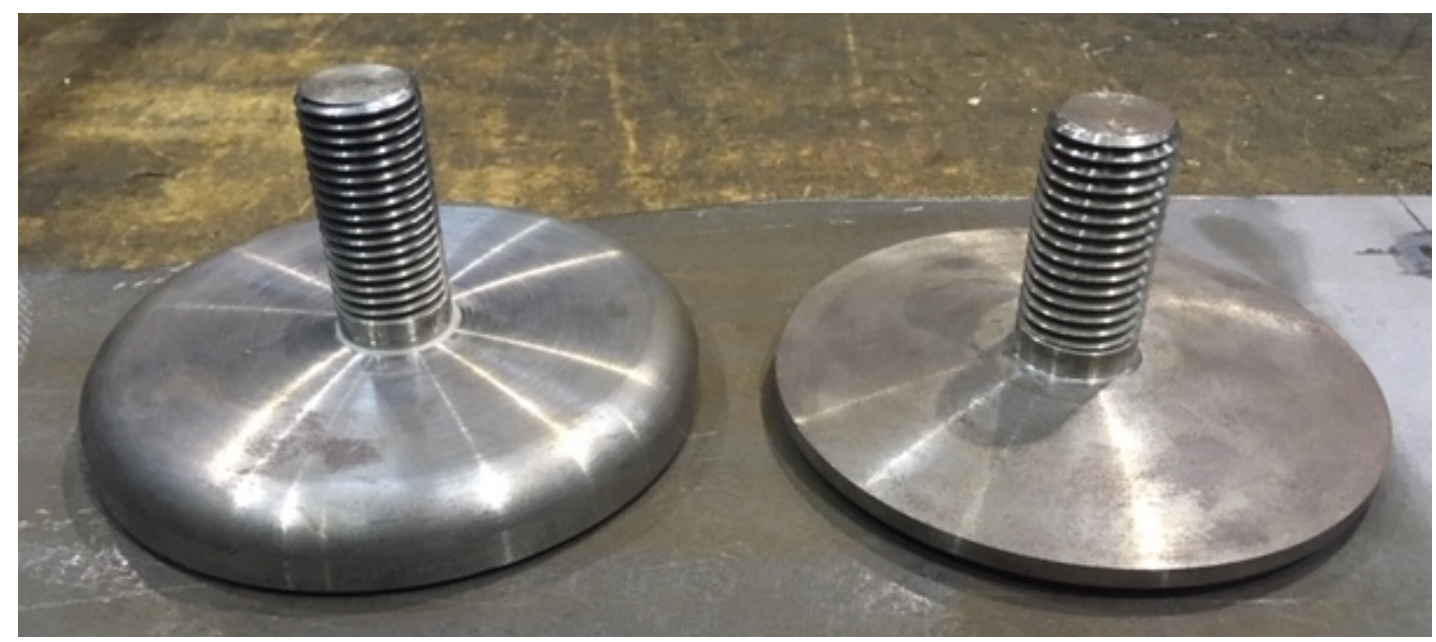

Fig. 4: Photo of studied C-Claw ${ }_{T M}$ steel fastener $T$ on the left and $S$ on the right. 

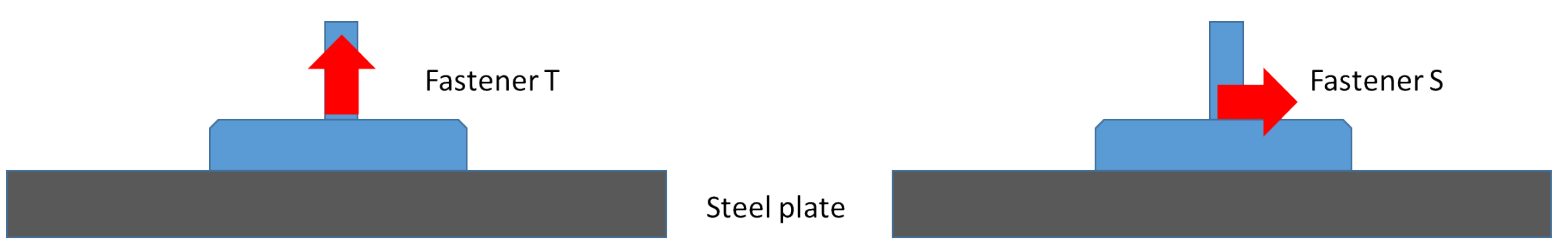

Fig. 5: Scheme of the two tested solicitation modes: Tension and Shear loadings. 


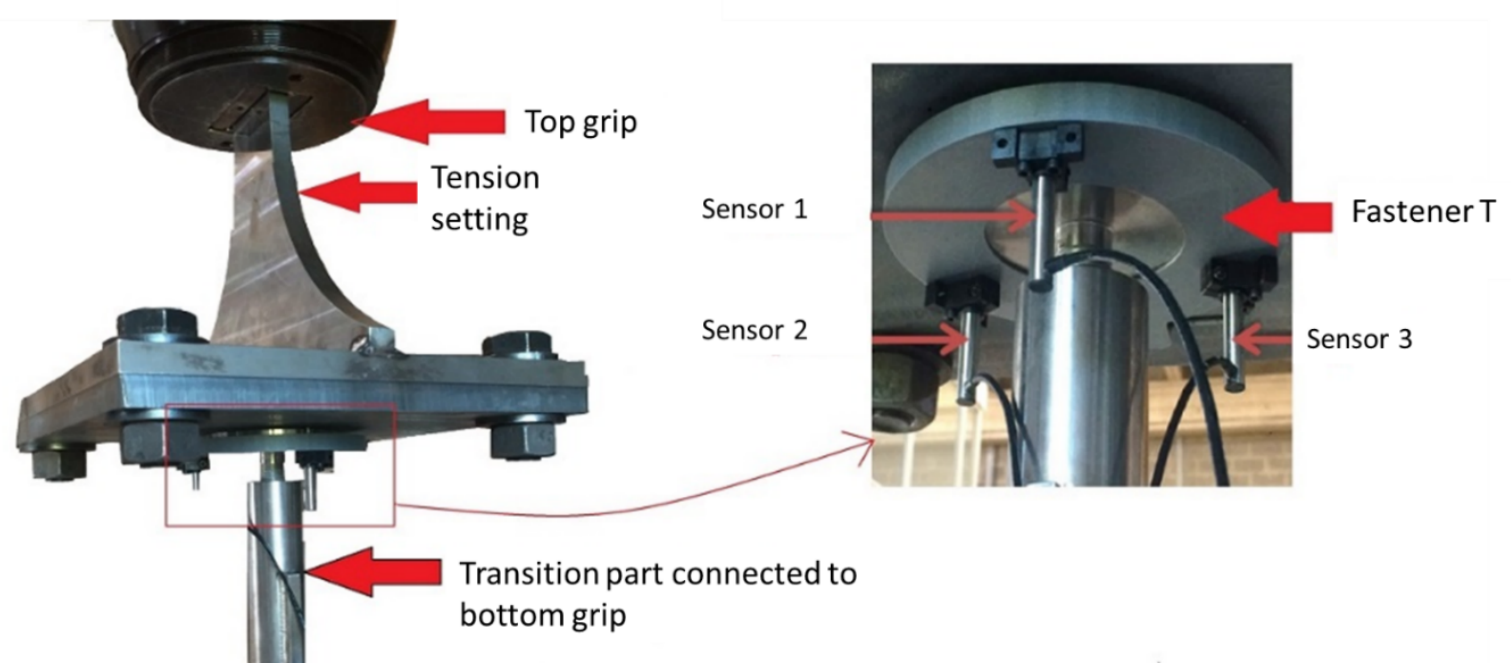

Fig. 6: Protocol of the tensile creep test. 


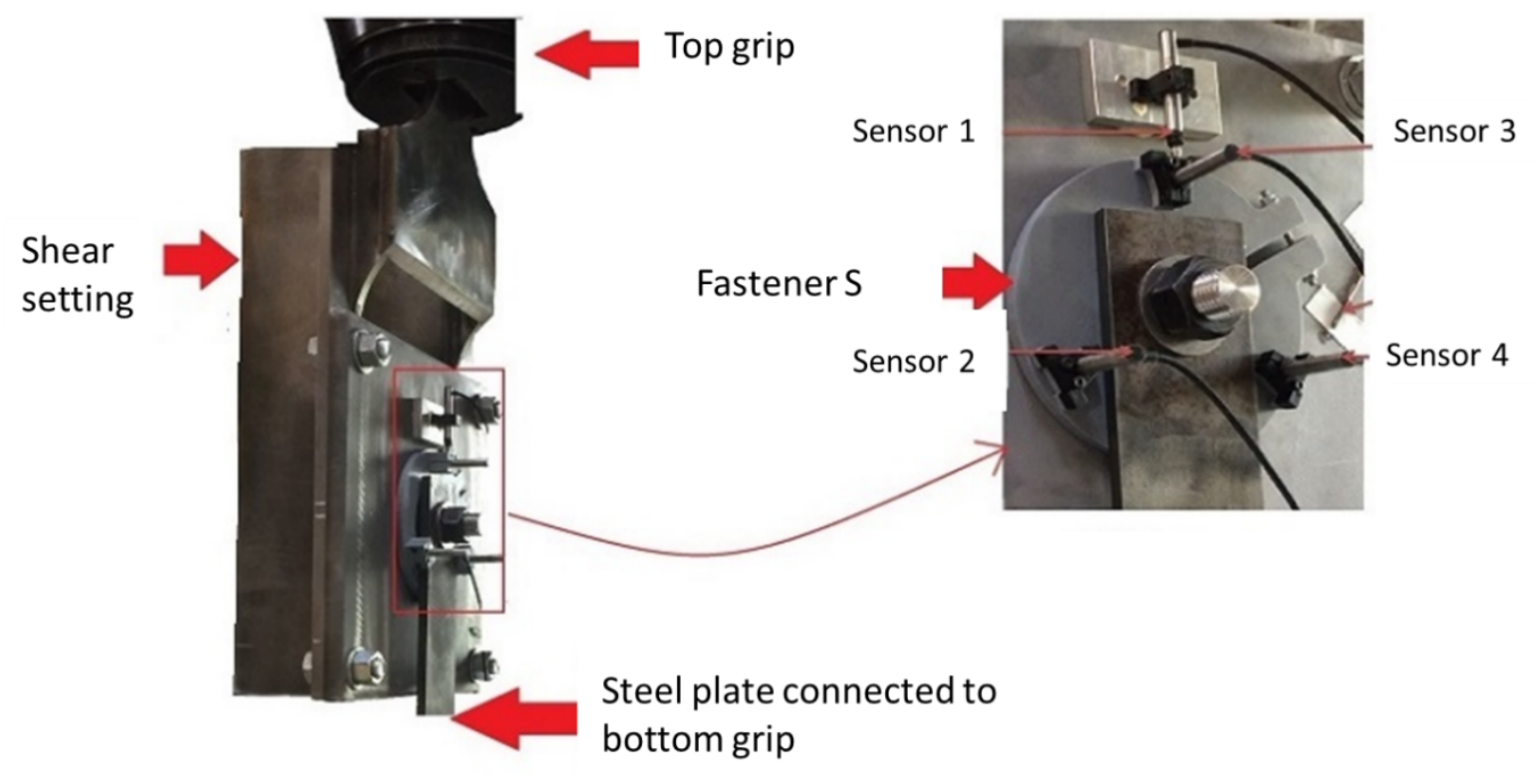

Fig. 7: Protocol of shear creep test. 


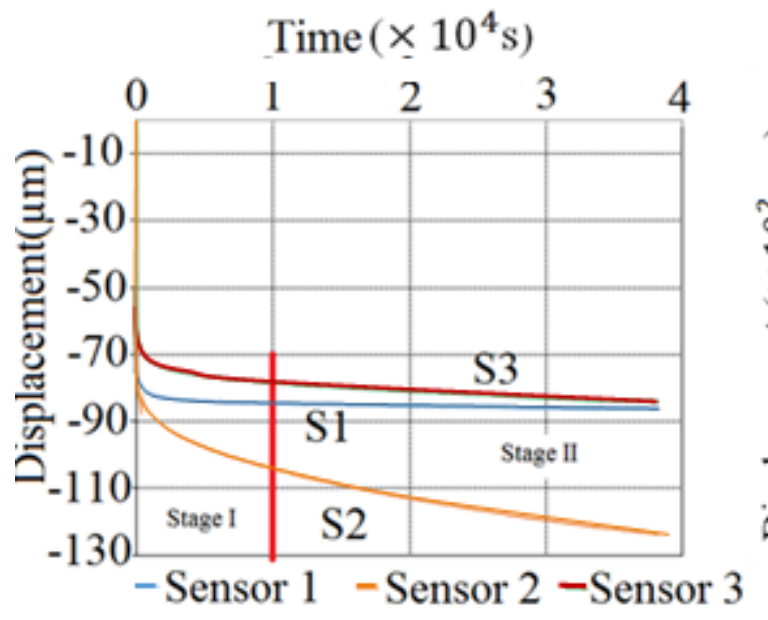

a) $59 \% \mathrm{~T}$ ult

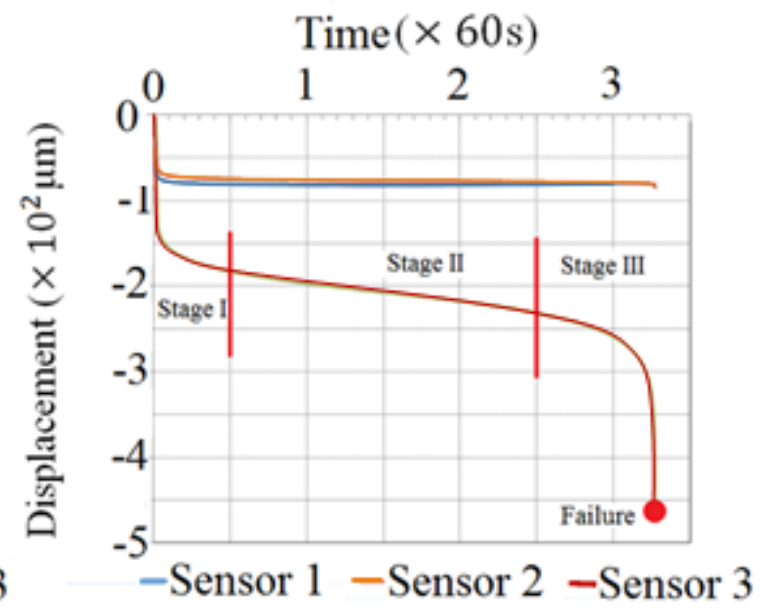

b) $69 \%$ T ult

Fig. 8: Measured displacements during tensile creep investigations at $\mathrm{T}=25^{\circ} \mathrm{C}$ 


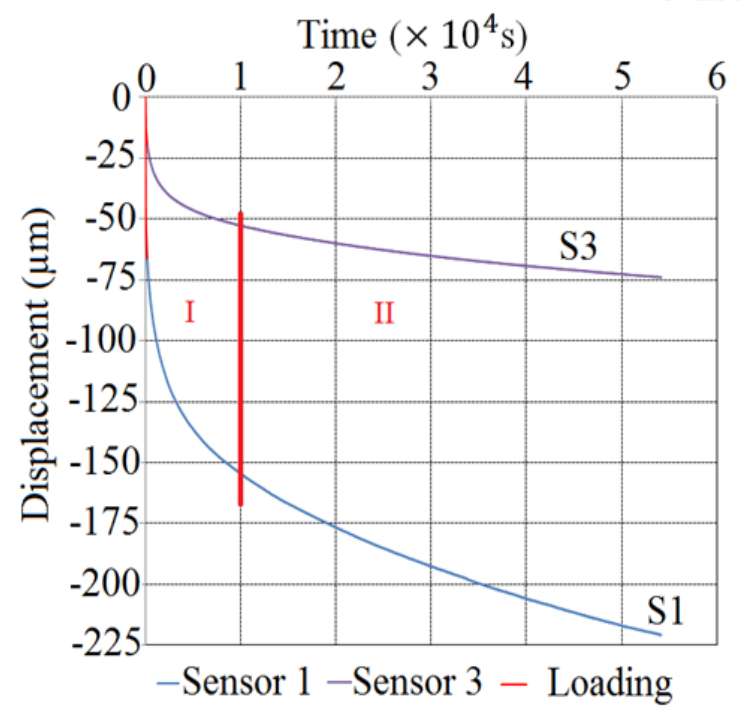

a) $59 \% \mathrm{~S}$ ult
Time $(\times 60 \mathrm{~s})$

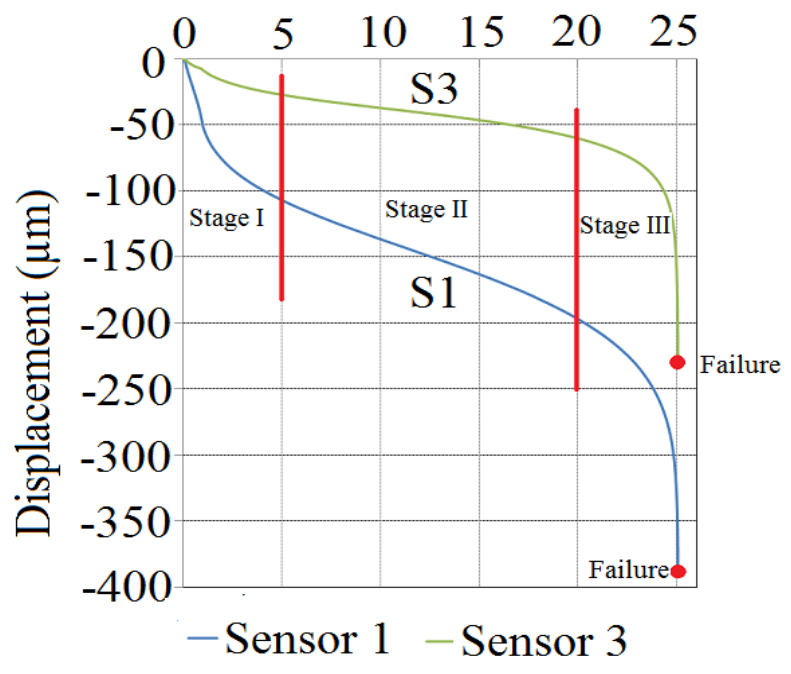

b) $67 \% \mathrm{~S}$ ult

Fig. 9: Creep shear at $\mathrm{T}=25^{\circ} \mathrm{C}$. 


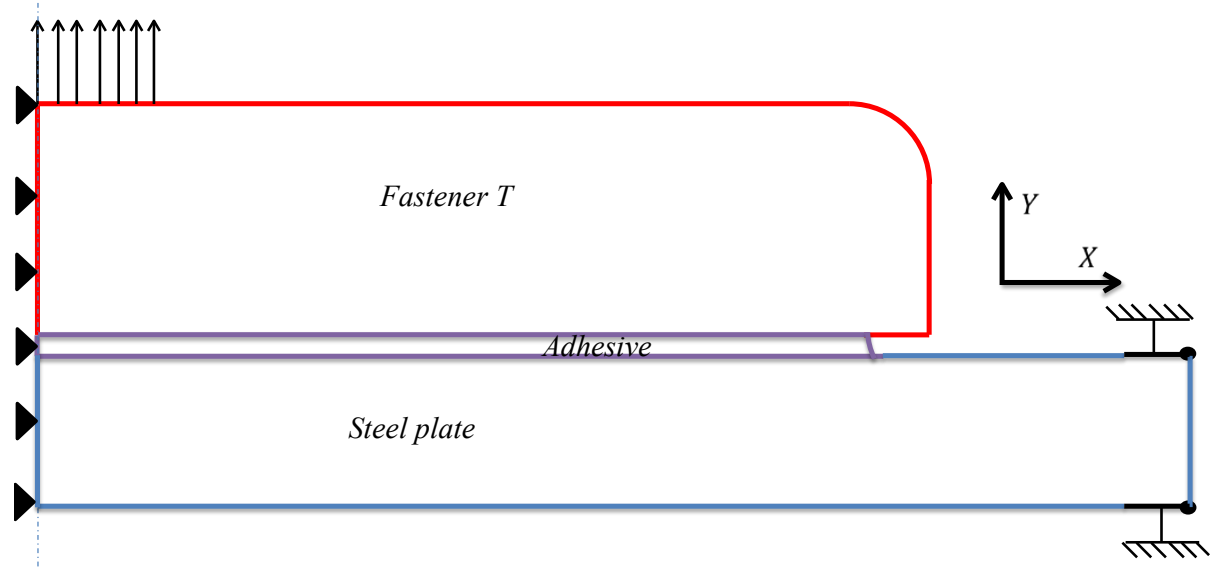

Fig. 10: Scheme of the studied 2D axisymmetric model. 


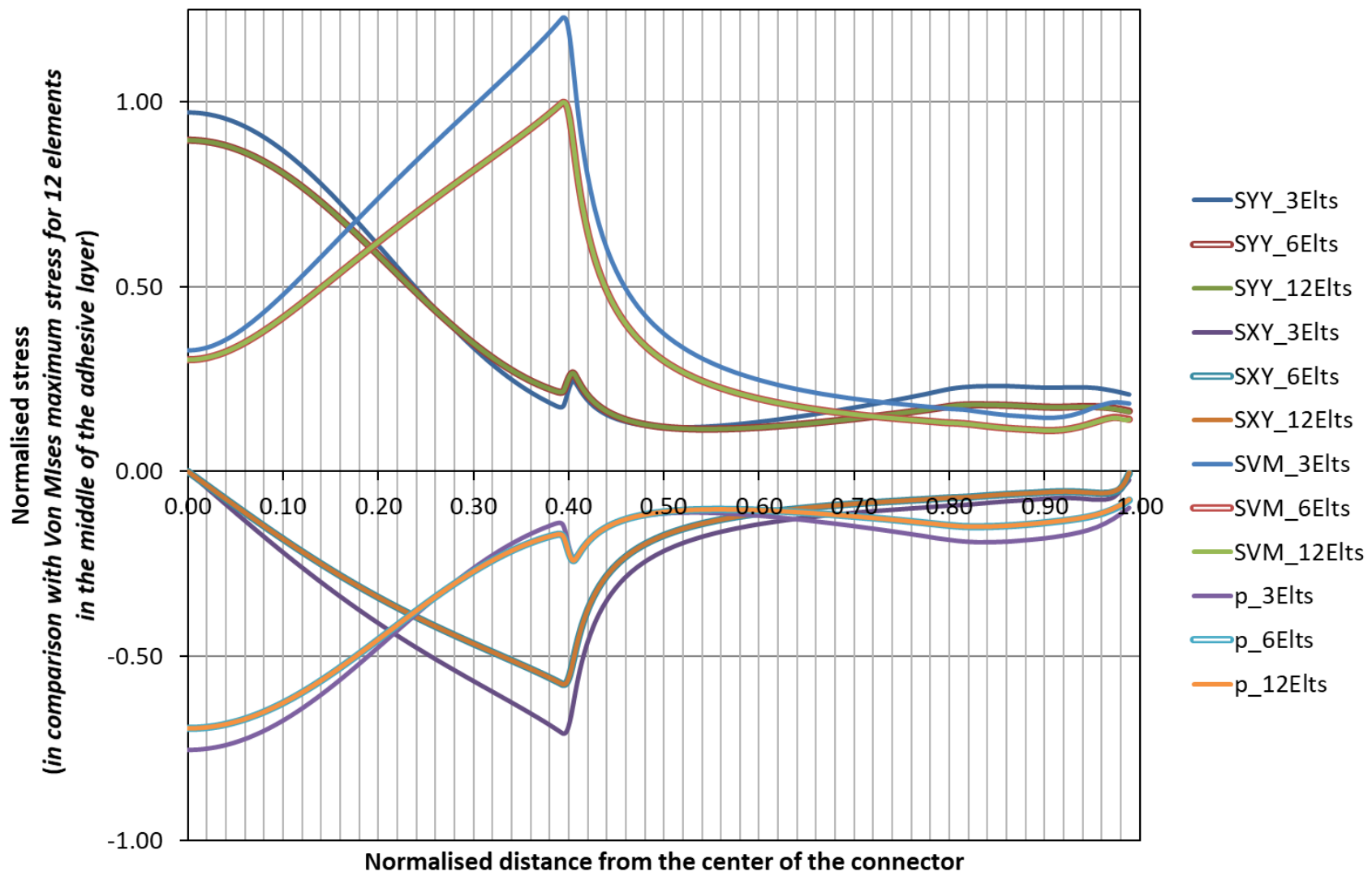

Fig. 11: Stress results obtained through finite element along the radius in the middle of the adhesive layer for different mesh sizes in tension 


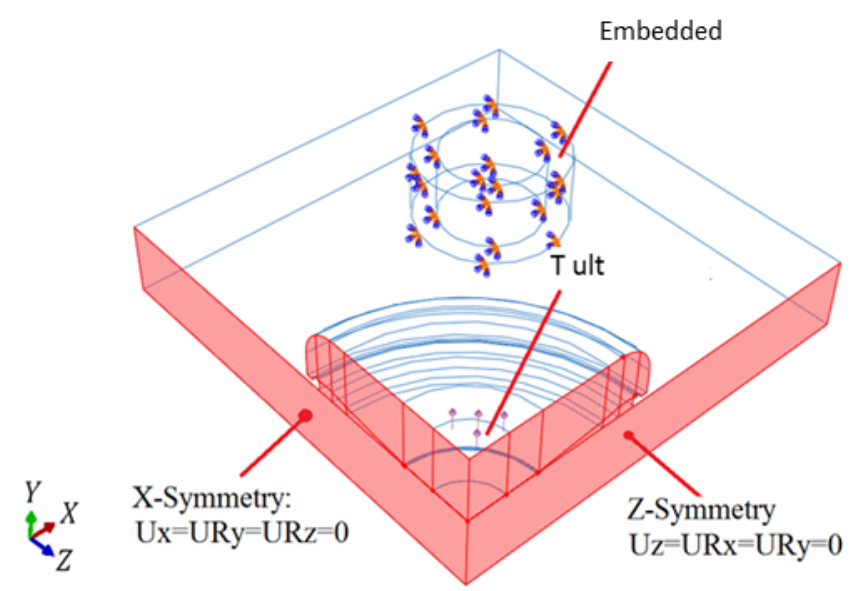

Fig. 12: 3D model in tension. 


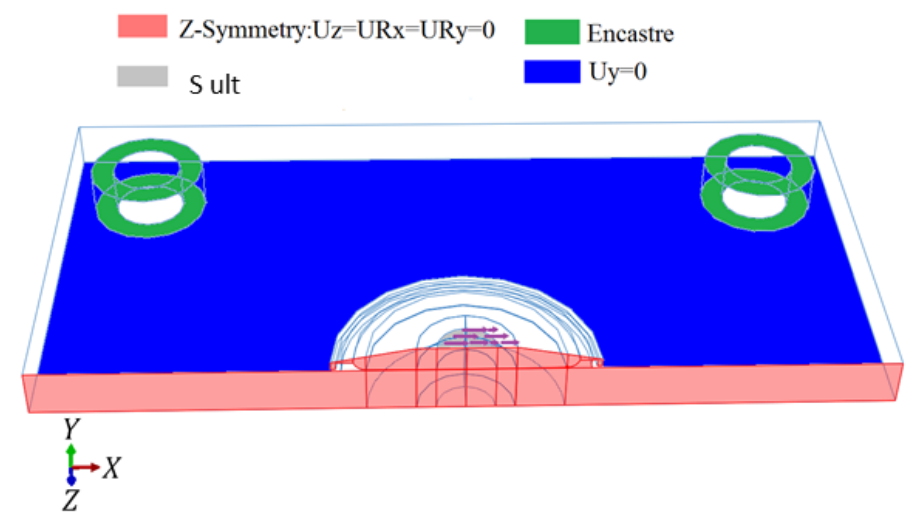

Fig. 13: Boundary conditions for the shear model. 


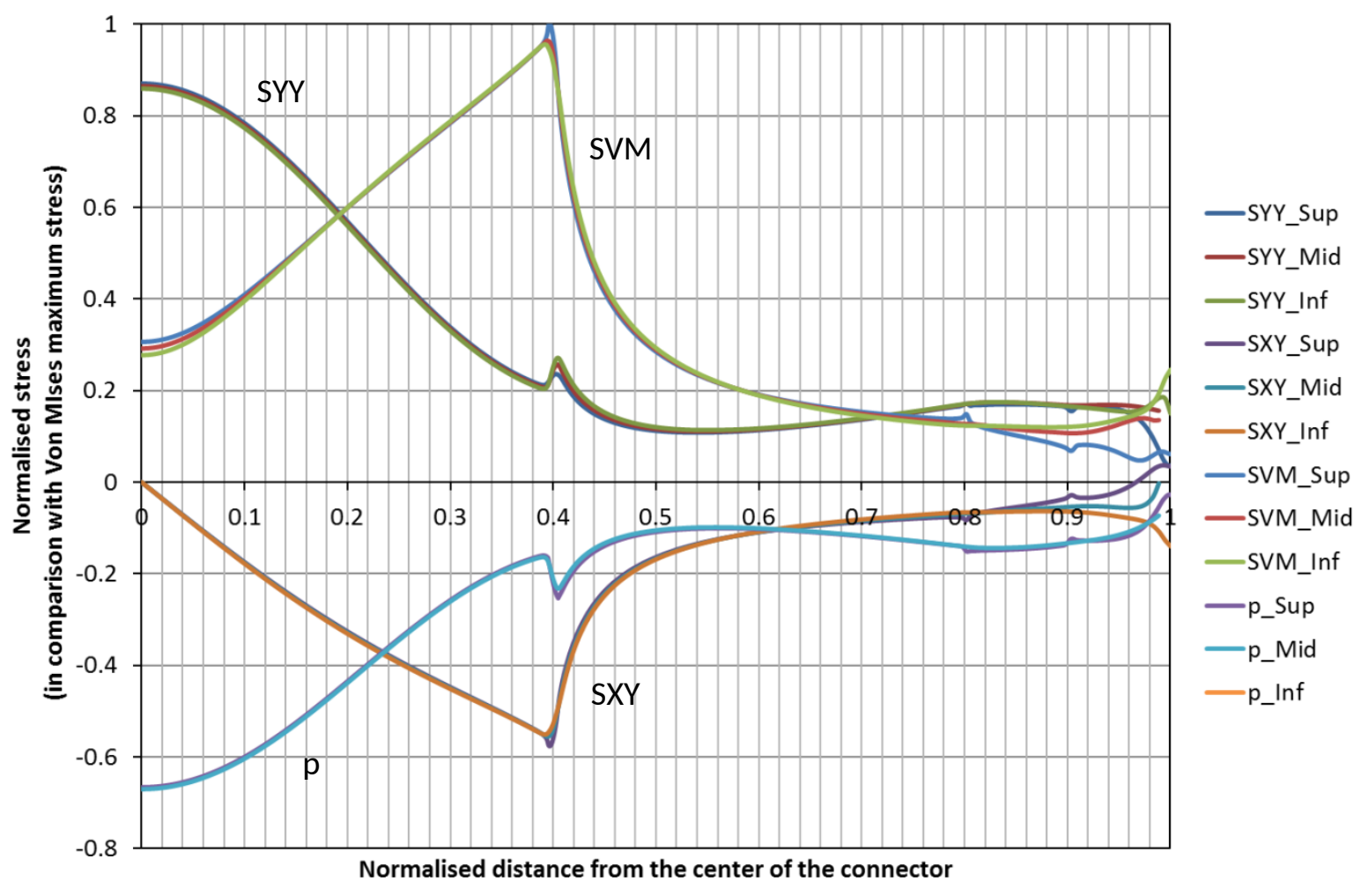

Fig. 14: Stress state for 2D axisymmetric model in tension. 


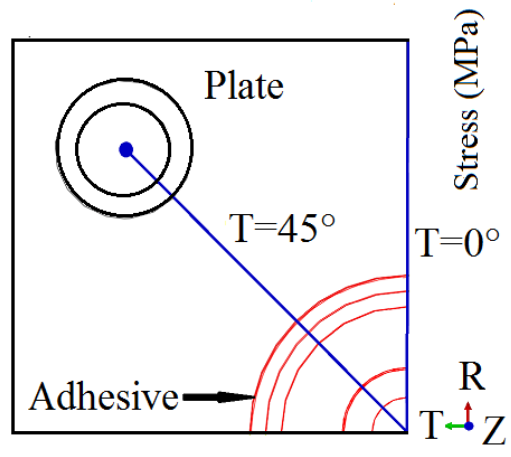

$\mathrm{R}(\mathrm{mm})$

a)

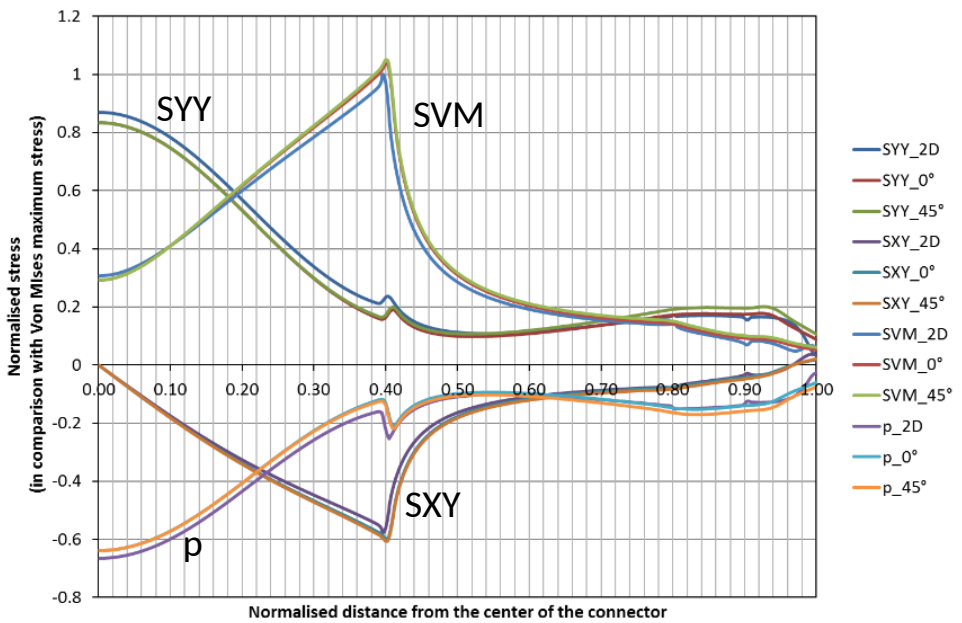

b)

Fig. 15: Comparison of the 2D and 3D model in tension. 


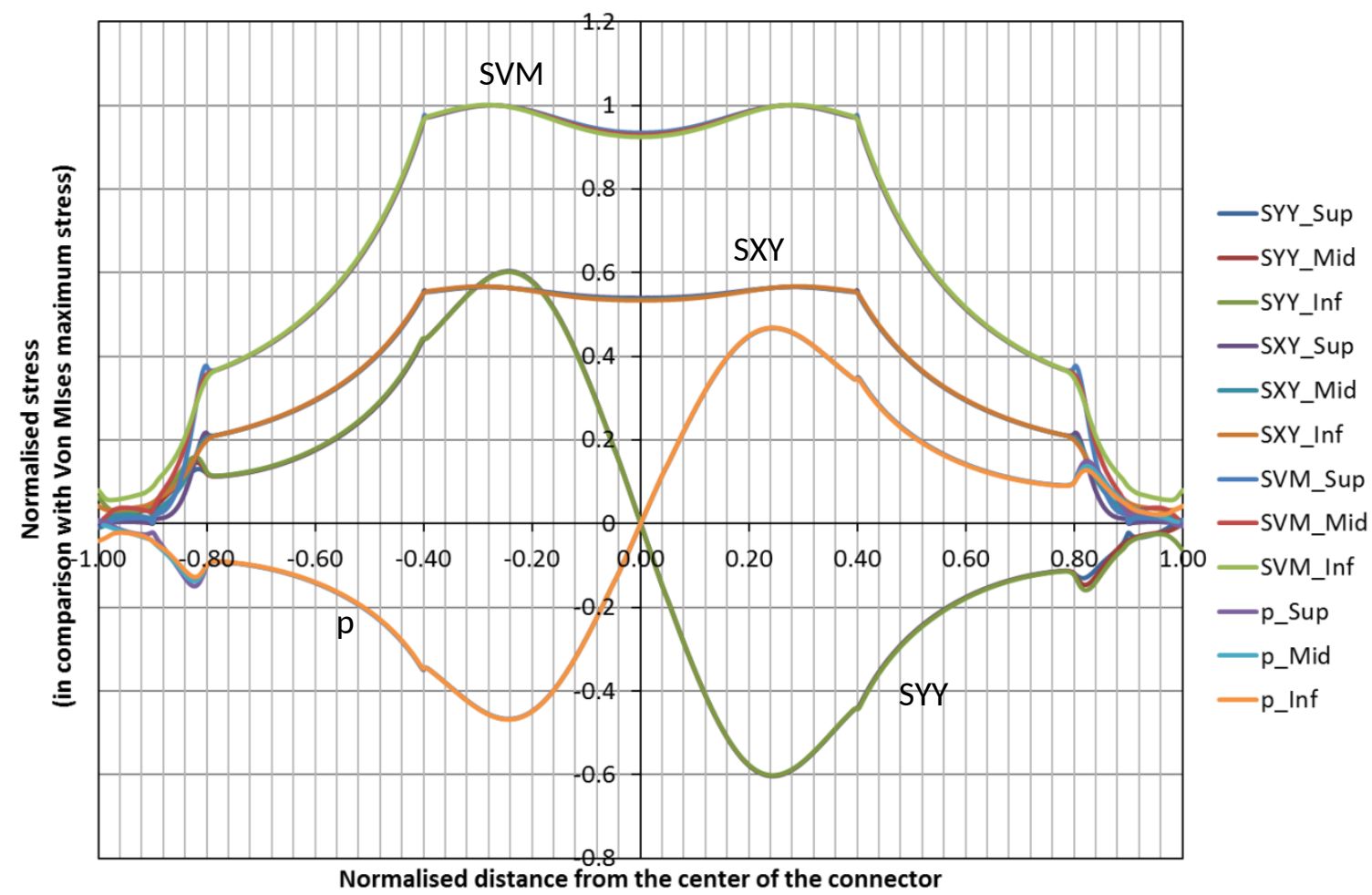

Fig. 16: Stress state along the adhesive for the shear model. 

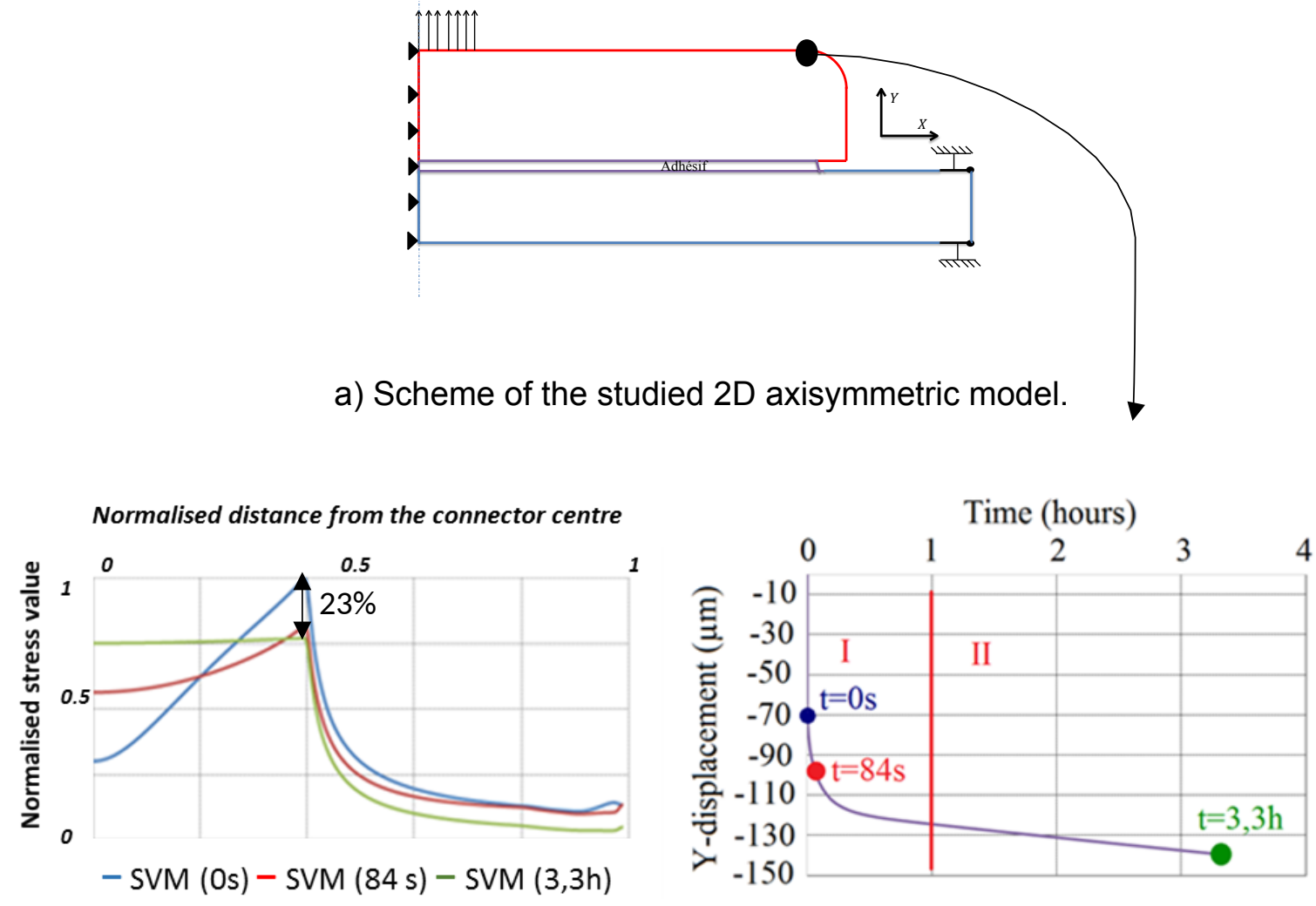

b) Stress redistribution

c) Creep displacement

Fig. 17: Redistribution of von Mises stress for tensile model. 


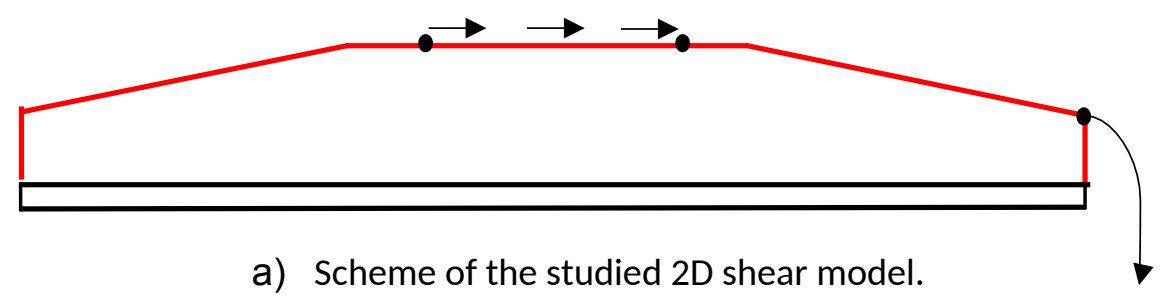

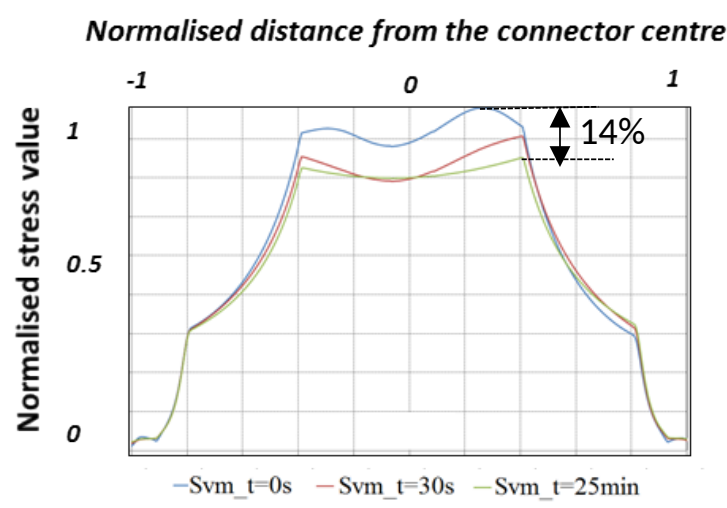

b) Stress redistribution

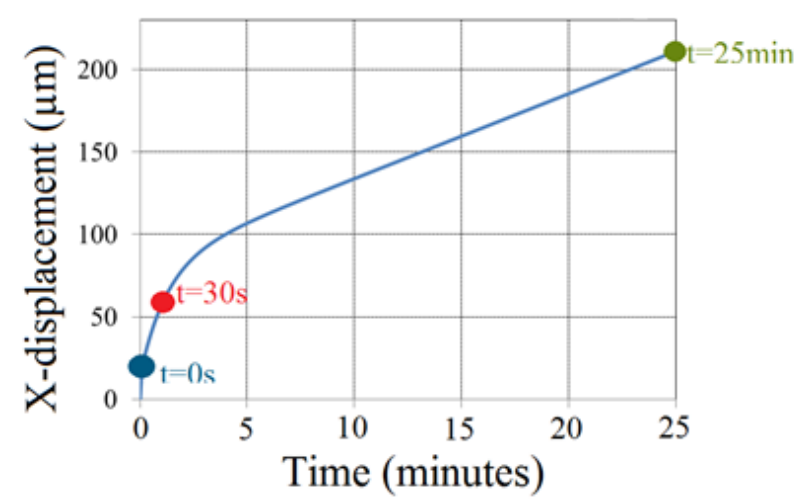

c) Creep displacement

Fig. 18: Redistribution of von Mises stress for the shear model. 


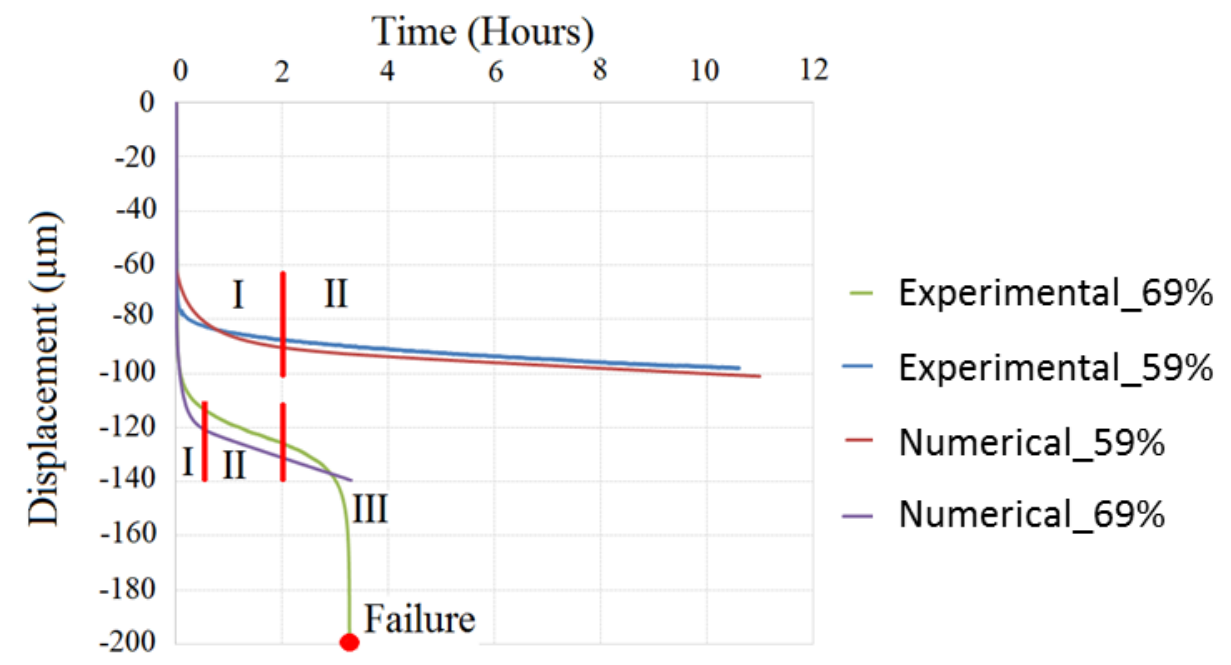

Fig. 19: Experimental and numerical comparison of out-of-plane displacement for tensile creep test at $25^{\circ} \mathrm{C}$. 


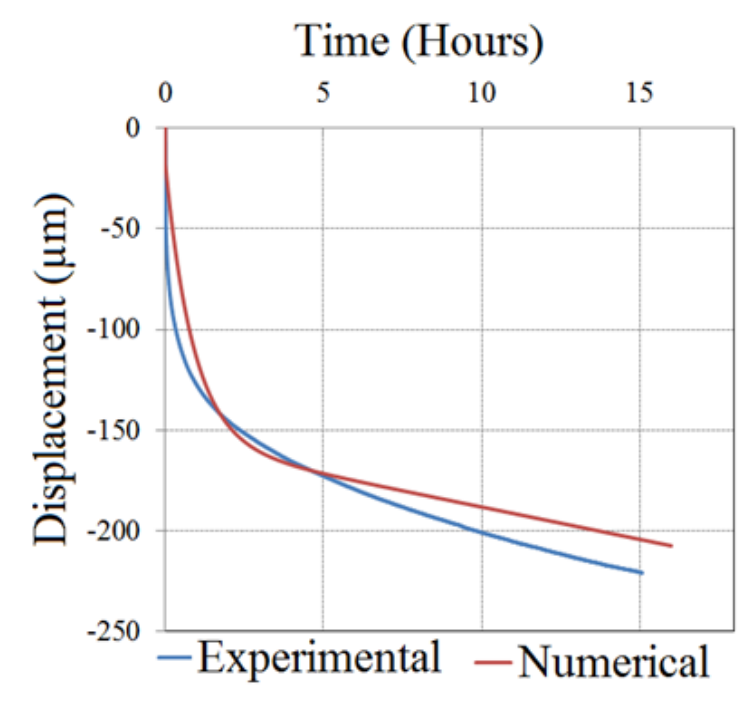

a) In-plane displacement (sensor 1)

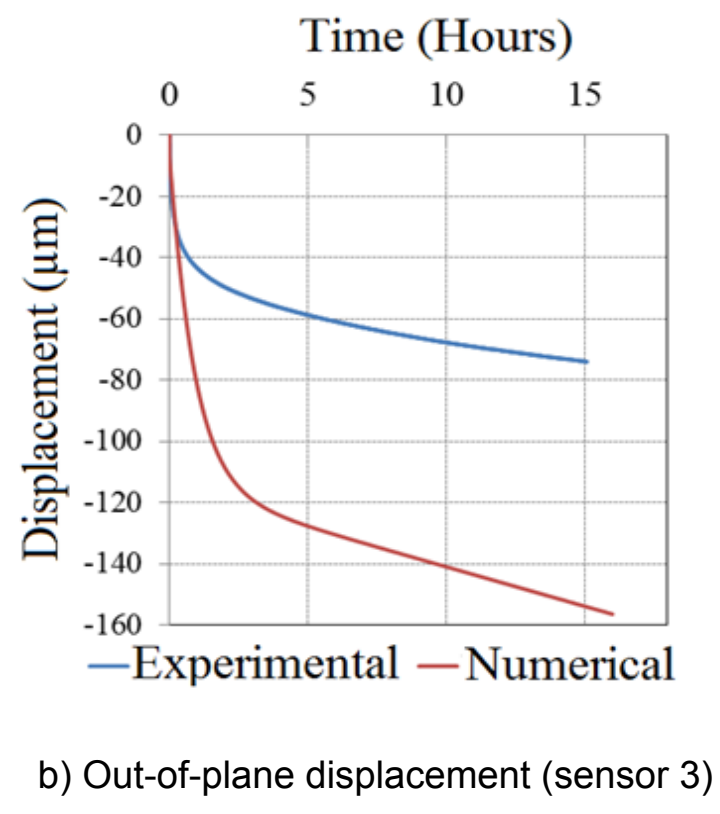

b) Out-of-plane displacement (sensor 3)

Fig. 20: Experimental and numerical comparison of creep displacements (in-plane and outof-plane) for shear test at $59 \%$ and $25^{\circ} \mathrm{C}$. 


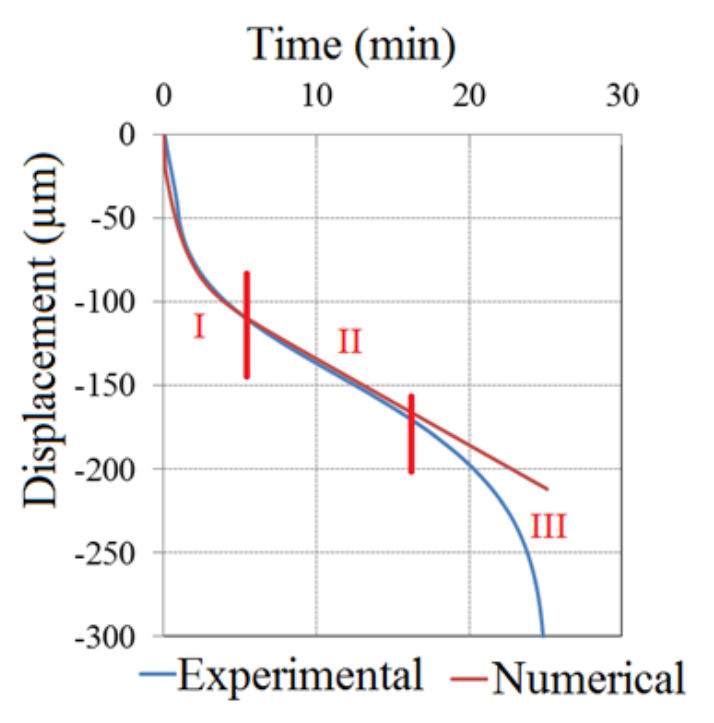

a) In-plane displacement (sensor 1)

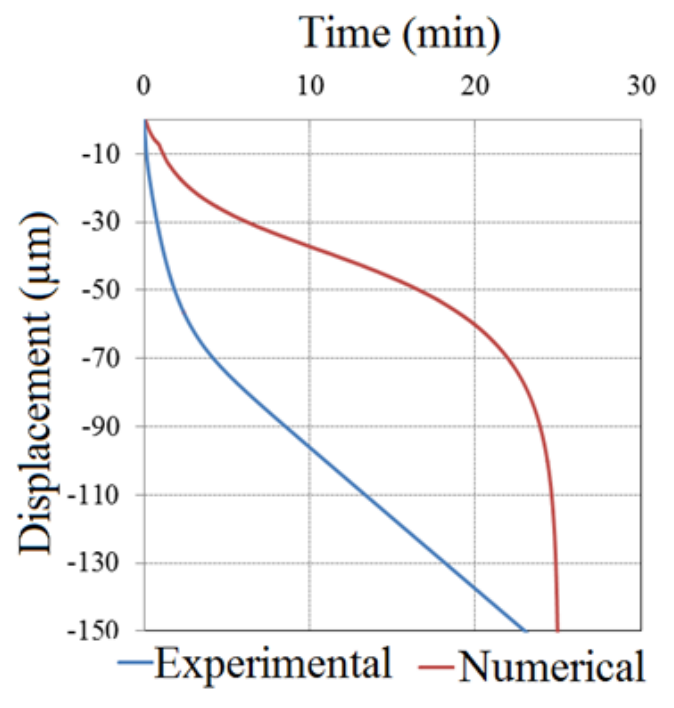

b) Out-of-plane displacement (sensor 3)

Fig. 21: Experimental and numerical comparison of creep displacements (in-plane and outof-plane) for shear test at $67 \%$ and $25^{\circ} \mathrm{C}$. 
Table 1: Characteristics of the tested adhesive provided by the supplier

\begin{tabular}{ccc}
\hline$E(\mathrm{MPa})$ & $R T(\mathrm{MPa})$ & $T g\left({ }^{\circ} \mathrm{C}\right)$ \\
\hline $1200-1700$ & $22-24$ & 80 \\
\hline
\end{tabular}

Table 2: Burger's law parameters

\begin{tabular}{lcccc}
\hline Applied load & $E_{1}(\mathrm{MPa})$ & $\eta_{1}(\mathrm{MPa} . \mathrm{h})$ & $E_{2}(\mathrm{MPa})$ & $\eta_{2}(\mathrm{MPa} . \mathrm{h})$ \\
\hline Tension (59\%) & 1200 & 5000 & 400 & 300 \\
Tension (69\%) & 1200 & 750 & 220 & 30 \\
Shear (59\%) & 1200 & 1700 & 40 & 35 \\
Shear (67\%) & 1200 & 19.83 & 100 & 2.083 \\
\hline
\end{tabular}

